\title{
Effect of the number of training trials on the event-related potential correlates of equivalence relations
}

\author{
Ryan Ríguel Barbosa do Espírito-Santo ${ }^{1} \cdot$ Gustavo Coelho Belleza Dias ${ }^{1} \cdot$ Renato Bortoloti $^{1}$. \\ Edson Massayuki Huziwara ${ }^{1}$
}

Published online: 11 September 2019

(C) The Psychonomic Society, Inc. 2019

\begin{abstract}
In the present study, we aimed to evaluate whether the number of training trials performed bythe participants during the baseline protocol in equivalence class experiments could modulatethe N400 evoked component. Two groups of 15 participants each followed a matching-tosampleprotocol to train on the conditional relations between four sets of abstract stimuli.Participants in the Extended Group performed approximately twice as many trials as those inthe Reduced Group. After having achieved the learning criteria in the equivalence tests,participants' neural activity was recorded during semantic judgment tasks that includedstimulus pairs of both the same (related pairs) and different (unrelated pairs) classes. Ourfindings indicate that participants in the Extended Group had similar N400 components forrelated and unrelated stimulus pairs. Conversely, participants in the Reduced Group had morenegative waveforms for unrelated stimulus pairs compared to the ones for related stimuluspairs. We discuss the necessity of a more careful analysis of the choice of the number ofbaseline training trials in experiments on equivalence relations and N400 component, anddraw parallels between our findings and the N400 effect previously described for high and low frequency words in the participants' native language.
\end{abstract}

Keywords equivalence relations $\cdot$ event-related potentials $\cdot \mathrm{N} 400 \cdot$ number of trials $\cdot$ college students

The stimulus equivalence paradigm (Sidman, 1994, 2000; Sidman \& Tailby, 1982) is an experimental model that provides a behavioral analytic treatment for human symbolic behaviors. It empirically distinguishes between symbolic learning and paired-associate learning, in which different stimuli enter into a relationship but do not replace each other as often occurs in genuine symbolic relations. According to this experimental paradigm, the symbolic relations can be functionally regarded as equivalence relations, defined in mathematics by the properties of reflexivity, symmetry, and transitivity. If a set of stimuli is related through equivalence, (a) the relations between each pair of stimuli in the set are symmetric or bidirectional (if $\mathrm{A}$ is related to $\mathrm{B}$, then $\mathrm{B}$ is related to $\mathrm{A}$ ); (b) the relations between two pairs with one stimulus in common are transitive (if A is related to B and B is related to C, then

Edson Massayuki Huziwara

huziwara.edson@gmail.com

1 Faculdade de Filosofia e Ciências Humanas, Departamento de Psicologia, Programa de Pós-Graduação em Psicologia: Cognição e Comportamento Humano, Universidade Federal de Minas Gerais (UFMG), Rua Flor de Esparódia, no. 51, apto. 203-Bloco 1, Bairro Ouro Preto, Belo Horizonte, Minas Gerais CEP: 31340 - 050, Brazil
$\mathrm{A}$ is related to $\mathrm{C}$ ); and (c) the relation between two exemplars of the same stimulus is reflexive (if $\mathrm{A}$ is related to $\mathrm{B}, \mathrm{A}$ is related to A and B is related to B). Different procedures, such as matching-to-sample (MTS; e.g., Sidman, 1971), stimulus pairing (Leader, Barnes, \& Smeets, 1996), or a go/no go procedure (Debert, Matos, \& Mcllvane, 2007) can be used to assess behavioral indicators of the logical properties of reflexivity, symmetry, and transitivity. These behavioral indicators are used to empirically distinguish between symbolic and nonsymbolic stimulus relations.

For the last five decades, equivalence relations have been documented in several studies with different populations and experimental conditions (e.g., Almeida-Verdu et al., 2008; Arntzen, 2012; de Rose, de Souza, Aiello, \& de Rose, 1992; Matos, Avanzi, \& McIlvane, 2006; Saunders \& McEntee, 2004; Saunders, Wachter, \& Spradlin, 1988; Sidman, 1994; Sidman \& Tailby, 1982; Tyndall, Roche, \& James, 2004). These studies demonstrate two important characteristics of symbolic behavior as defined by the stimulus equivalence paradigm: (a) new conditional relations emerge from those previously trained, and (b) physically different stimuli become substitutable for the control of behavior. In addition, equivalence class formation also enables the occurrence of the derived transfer of function. Specifically, following the 
establishment of equivalence classes, the behavioral function exerted by (or established for) one of its members can be transferred to other members of the class without additional training (Y. Barnes-Homes, Barnes-Holmes, Smeets, \& Luciano, 2004; Bortoloti \& de Rose, 2007, 2009; Smeets \& Barnes-Holmes, 2003). This is consistent with the idea that in many cases one reacts to symbols the same way one would react to the events they referred to. Thus, a stimulus that has (or acquires) certain functions can be considered as a referent, and stimuli equivalent to it can be referred to as symbols capable of replacing it in some contexts.

In recent years, a range of different procedures combined with standard MTS trials has been used for assessing issues related to the equivalence class formation. Studies involving semantic differential ratings (Bortoloti \& de Rose, 2009), lexical decision tasks (D. Barnes-Holmes et al., 2005; Bortoloti $\&$ de Rose, 2011), the Implicit Association Test (O’Toole, Barnes-Holmes, \& Smyth, 2007), the Implicit Relational Assessment Procedure (Bortoloti \& de Rose, 2012), and, of special importance for the current study, the event-related potential (ERP) technique (D. Barnes-Holmes et al., 2005; Bortoloti, Pimentel, \& de Rose, 2014; Granerud-Dunvoll, Arntzen, Elvsåshagen, Hatlestad-Hall, \& Malt, 2019; Haimson, Wilkinson, Rosenquist, Ouimet, \& McIlvane, 2009; Tabullo, Yorio, Zanutto, \& Wainselboin, 2015a, b), serve as examples. The ERP technique has permitted, for instance, the electrophysiological investigation of the functional overlap between semantic and equivalence relations by means of extracting the N400 brainwave component.

The ERP technique consists of placing arrays of electrodes on the participant's scalp to measure the electrical activity from groups of cortical neurons while he or she performs a given activity. The neuronal activity of interest occurs primarily from the onset of a specific environmental event (e.g., presentation of a stimulus) to approximately $1,000 \mathrm{~ms}$ after that event. The neuronal activity evoked over this time span varies in amplitude, strength, and frequency, which can all be graphically represented by positive or negative wavelengths (Kutas \& Federmeier, 2011; Lau, Phillips, \& Poeppel, 2008; Luck \& Kappenman, 2011). To date, the ERP considered to be the "brainwave marker of semantics processing" (cf. Hajra et al., 2018) is the N400, a negative-going wave that peaks at approximately $400 \mathrm{~ms}$ following the stimulus-stimulus pairing presentation. In an experimental setup with a series of semantically related word pairs (e.g., chair-table) and unrelated word pairs (e.g., chair-lion), an across-trial comparison of ERP signals shows a clear differentiation in the range of the N400 evoked by related and unrelated word pairs. Namely, large negative-going wave are evoked by unrelated words while small negative-going wave are evoked by related words. This difference in the size of the wave is a dependent measure of meaning comprehension (Holcomb 1993; Kutas \& Hillyard, 1984, 1989).
The ERP technique has recently been used to verify whether related and unrelated stimulus pairs in equivalence class experiments yield analogous N400 components (e.g., Bortoloti et al., 2014; Granerud-Dunvoll et al., 2019; Haimson et al., 2009; Tabullo et al., 2015a, b). Using nonsense words as stimuli, D. Barnes-Holmes et al. (2005, Experiment 3) established conditional relations $\mathrm{AB}$ (A1B1 and $\mathrm{A} 2 \mathrm{~B} 2$ ) and $\mathrm{AC}$ ( $\mathrm{A} 1 \mathrm{C} 1$ and $\mathrm{A} 2 \mathrm{C} 2$ ). The participants were given trials designed to probe for both symmetry (B1A1, $\mathrm{B} 2 \mathrm{~A} 2, \mathrm{C} 1 \mathrm{~A} 1$, and $\mathrm{C} 2 \mathrm{~A} 2)$ and transitivity $(\mathrm{B} 1 \mathrm{C} 1, \mathrm{~B} 2 \mathrm{C} 2$, $\mathrm{C} 1 \mathrm{~B} 1$, and $\mathrm{C} 2 \mathrm{~B} 2$ ) relations. Finally, the neuronal activity was recorded while participants performed semantic judgment tasks with the nonsense words previously used in the equivalence class procedure. The judgment task presented pairs of related stimuli (i.e., A1-B1, C2-A2, B1-C1, etc.) and pairs of unrelated stimuli (i.e., A1-B2, C2-A1, B1-C2, etc.) in a random order. Similar to the meaningful words, presentation of an unrelated pair (e.g., B1C2) evoked greater negativity around $400 \mathrm{~ms}$ when compared with the presentation of a related pair (e.g., B1C1).

On the one hand, an increasing number of studies indicate that the derived relations in equivalence class experiments and the semantic relations in the natural language evoke similar patterns of neural activity (e.g., D. Barnes-Holmes et al., 2005; Bortoloti et al., 2014; Granerud-Dunvoll et al., 2019; Haimson et al., 2009; Tabullo et al., 2015a, b). This suggests that the stimulus equivalence paradigm may be a useful experimental model of semantic relations in naturally occurring language. On the other hand, however, some these experiments have inconsistent results. For instance, in Haimson et al. (2009), participants were given an MTS training protocol to establish conditional relations between nonsense words and abstract visual stimuli. Next, some participants were first exposed to equivalence test trials using the MTS protocol and then to semantic judgment tasks in which neuronal activity was recorded (G1). Other participants were exposed to the semantic judgment tasks followed by equivalence test trials (G2). G1 participants had a clear differentiation in the N400 evoked by related or unrelated stimulus pairs after the first set of trials, while G2 participants exhibited a clear differentiation in the N400 only in the last portion of trials. According to the authors, testing was necessary to establish the context for the emergence of equivalence relations. If this is true, equivalence classes were established during the MTS tests, and the N400 was recorded in the semantic judgment tasks for the G1 participants. For G2 participants, the establishment of the classes and the N400 recording occurred simultaneously during the semantic judgment tasks, which is why the N400 differentiation was delayed. Interestingly, five other studies evaluated neuronal activity related to equivalence class formation without previous MTS test and, as opposed to Haimson et al., two of those studies clearly showed the N400 effect (Tabullo et al., 2015a, b), while three had 
ambiguous results (Menendéz et al., 2018; Wang \& Dymond, 2013; Yorio, Tabullo, Wainselboim, Barttfeld, \& Segura, 2008; for a review, see Silveira et al., 2019).

It is noteworthy that even though these experiments recorded N400 without previous MTS tests, the experimental protocols differed from one another in many important parameters such as training procedures (i.e., simultaneous vs. delayed MTS), number of formed classes, class size, and nature of the stimuli (i.e., pseudowords, abstract forms, or both). Although previous MTS tests were related to better N400 results, other training parameters could have modulated the ERP. A deeper understanding of the role of the ERPs requires parametric research to describe in detail the modulating effect of the parameters used in equivalence relation experiments on the N400 component.

The current study aimed to advance the electrophysiological investigation of the functional overlap between semantic and equivalence relations by assessing methodological parameters of class formation that may influence the strength of the N400 component. Specifically, we evaluated the modulatory effects of the number of baseline training trials. In standard MTS preparations, participants are exposed to as many trials as needed for achieving the learning criterion, leading to variation in the number of trials performed by each participant at baseline. The effect of such variation in the N400 component remains unclear; however, it appears to be irrelevant in most experiments related to equivalence class formation.

Moreover, recent studies indicate that a higher number of training trials is associated with a better performance on both equivalence (Espírito-Santo, Rico, \& Huziwara, 2019; Straatmann, Almeida, \& de Rose, 2014) and derived transfer of function tests (Bortoloti, Rodrigues, Cortez, Pimentel, \& de Rose, 2013). For instance, Bortoloti et al. (2013) evaluated the degree of the derived transfer function between faces expressing emotions (i.e., happiness, sadness, and anger) and abstract stimuli, measured by means of a semantic differential instrument, in college students exposed to different numbers of training trials. Participants assigned to the overtraining group performed twice as many trials as participants assigned to the regular group. The results showed that the overtraining group had a higher level of derived transfer function than the regular group.

Even considering that such a variable was not directly evaluated by ERP researchers in previous experiments, we tried to describe ERP patterns that could be expected based on experiments that evaluated N400, using words from the participants' natural language. Moreno and Kutas (2005), for instance, demonstrated that language proficiency produces temporal modulations in the N400 component. In particular, the N400 component was found to be significantly later for second language in comparison to that of first language stimuli, when bilingual participants were exposed to semantic judgment tasks. If we consider that the number of training trials improves the relational strength among equivalent stimuli, this parameter may improve the participants" "proficiency" in the classes, resulting in N400 latency differences (i.e., the higher the number of training trials, the shorter the latency of the N400; Bortoloti et al., 2014).

In addition, the N400 component seems to be negatively modulated by the frequency in which certain pairs of words are presented together throughout the experiment (Kutas \& Federmeier, 2000; Smith \& Halgren, 1987). In other words, after several presentations of the same unrelated word pair (e.g., chair-lion), this specific pair would evoke a small negative-going wave. Thus, if such a repetition effect occurs in the present experiment, a higher number of training trials would decrease the amplitude of the N400 component, even for unrelated stimulus pairs. In this sense, the overexposure to training trials could evoke reduced negativity within the N400 component.

To evaluate this issue, a group of 15 participants was exposed to a baseline training with approximately 400 trials, while a second group of 15 participants was exposed to a baseline training with approximately 200 trials. The ERP recordings were made after the equivalence class formation and an across-group comparison was made to relate the modulations in the N400 wavelengths to a prior exposure to a different number of baseline training trials.

\section{Method}

\section{Participants}

Thirty undergraduate students including 18 women and 12 men, ranging in age from 18 to 30 years, took part in this study. An absence of previous exposure to the experimental behavior analysis concepts was a requirement for participation. Participants were randomly assigned to one of two groups: the extended-number-of-baseline trials (extended group) and the reduced-number-of-baseline trials (reduced group).

The participants read and signed the informed consent terms, and the procedures performed in this experiment were in accordance with the Brazilian Health Committee. In addition, the project was scrutinized by the Ethics Committee of the Federal University of Minas Gerais (UFMG). The information in the informed consent was provided in Brazilian Portuguese.

\section{Setting and equipment}

The experiments were conducted in a laboratory at a Brazilian federal institution of higher education. A notebook running on the EAM 4.3 software (Capobianco \& 


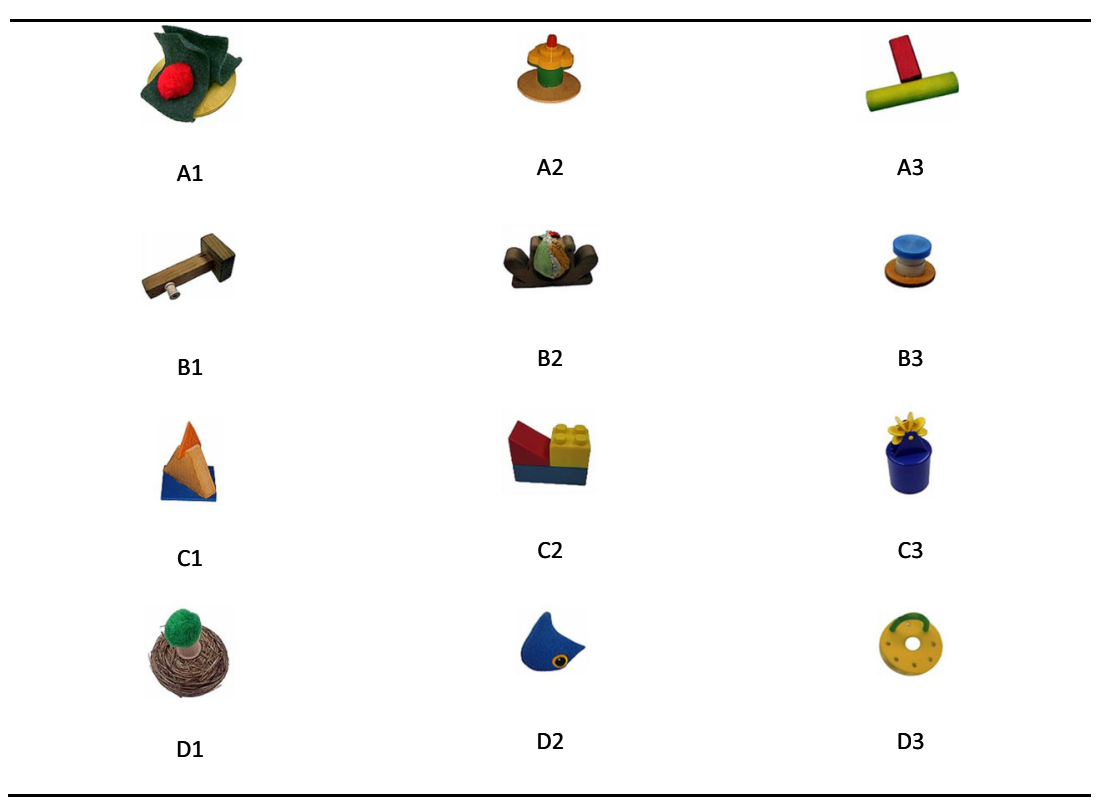

Fig. 1 Stimuli used in the study and their respective alphanumeric labels. Labels were not presented to the participants

Picanço, 2015) was used to present the baseline and emergent relations test trials, to record the participants' responses, and to provide feedback on the correct and incorrect choices.

A second computer was used for the semantic judgment tasks and to conduct the electrophysiological recordings. The Neurosoft Neuron-Spectrum 4/EPM equipment signal amplifier and the Neuron-Spectrum.NET software were used to capture and record the ERPs. The room was acoustically isolated so as to ensure a better quality of electrophysiological recordings.

\section{Stimuli}

Twelve abstract figures were randomly distributed into four sets-A, B, C, and D (see Fig. 1). Participants established three equivalence classes, each formed by four stimuli (i.e., A1B1C1D1, A2B2C2D2, and A3B3C3D3), after the baseline training.

\section{Procedure}

Baseline relations training Baseline relations were trained using a zero-delay MTS procedure. All trials started with a sample stimulus being displayed in one of nine possible quadrants on a computer screen. Clicking on the sample caused it to disappear, and after a delay of $0 \mathrm{~s}$, three comparison stimuli were presented in three of the remaining eight quadrants. The position of the sample and the comparison stimuli varied nonsystematically throughout the trials. To avoid establishing an unprogrammed bias in stimulus control, the positions of the comparison stimuli were balanced so that the correct comparisons were presented for the same number of times in each position.

Participants had to choose one of the three comparisons, and clicking on the correct one produced a point, which was displayed during the $1.5 \mathrm{~s}$ intertrial interval (ITI). Points were added to a counter whenever a correct choice was made and accumulated over the training trials. In contrast, clicking on one of the incorrect comparisons produced only the ITI. It should be noted that these points only provided an index of the participant's performance in some sessions during the training procedure and were not exchanged for other reinforcers.

Considering the specific aim of the present experiment (i.e., to evaluate the effect of the number of trials completed at baseline on the N400 component), the accuracy-based learning criterion was not used, as this criterion allows for variation in the number of baseline trials across participants. Instead, all participants were exposed to each block only once throughout the training steps. Due to this restriction, prompts were used in the initial trials of each conditional relation at the baseline training to enhance learning. In the trials with prompts, the phrase "When this is here" (written in Portuguese) appeared above the sample, whereas the phrase "Choose this" (written in Portuguese) appeared above the correct comparison.

Participants assigned to the extended group started the procedure by training on the relations between the A and B stimulus sets, called AB training. For this, they were exposed to a training block consisting of 12 trials with prompts and 48 noninstructed trials. Then, the participants progressed to the AC training, also composed of 12 trials with prompts and 48 noninstructed trials. Finally, a block with 48 noninstructed and 
intermixed $\mathrm{AB}$ and $\mathrm{AC}$ trials (Cumulative Baseline $\mathrm{I}$ ) was presented upon completion of the $\mathrm{AC}$ training.

The AD training, which followed the Cumulative Baseline I, used the same number of trials with prompts and no instructions (i.e., 12 and 48, respectively). Upon completion of the $\mathrm{AD}$ training, the Cumulative Baseline II consisting of $48 \mathrm{AD}$ noninstructed trials, $24 \mathrm{AB}$ noninstructed trials, and $24 \mathrm{AC}$ noninstructed trials was administered. Since the AD relations were not presented in the Cumulative Baseline I, different number of trials for each relation were used to balance the number of trials per relation. Finally, upon completion of the Cumulative Baseline II, participants were exposed to a block of 72 noninstructed trials consisting of equal proportions of the $\mathrm{AB}, \mathrm{AC}$, and $\mathrm{AD}$ relations (Cumulative Baseline III). Points were no longer given for correct responses during Cumulative Baseline III in preparation for the unreinforced trials performed in the test sessions.

Participants in the reduced group followed the same sequence of training steps as the participants in the extended group, except they were exposed to fewer baseline trials in the training phase. The $\mathrm{AB}, \mathrm{AC}$, and $\mathrm{AD}$ blocks for the reduced group comprised 12 trials with prompts and 24 noninstructed trials. In addition, the Cumulative Baseline I, Cumulative Baseline II, and Cumulative Baseline III blocks consisted of 24 (12 AB and $12 \mathrm{AC}), 48$ (12 AB, $12 \mathrm{AC}$, and $24 \mathrm{AD})$, and $36(12 \mathrm{AB}, 12 \mathrm{AC}$, and $12 \mathrm{AD})$ noninstructed intermixed trials, respectively. To summarize, participants assigned to the Extended group were exposed to 396 baseline trials while participants assigned to the reduced group were exposed to 216 baseline trials.

Equivalence test The equivalence test assessed the emergence of $\mathrm{BC}$ and $\mathrm{CB}$ relations. The test consisted of 24 trials (12 BC and $12 \mathrm{CB}$ ) without feedback for both groups. Participants who achieved $85 \%$ of correct responses in this test were transferred to the semantic judgment task.

Semantic judgment task Prior to the beginning the semantic judgment task, the electrodes were placed on the participants' scalp for neural activity recording. Participants from both groups were exposed to the same proportion of trials in the semantic judgment task. The first 48-trial block presented BC and $\mathrm{CB}$ equivalent and nonequivalent pairs. The related (e.g., B1C1, C2B2) and unrelated (e.g., B1C2, C2B1) pairs appeared in equal proportion in this block. Next, participants were exposed to a 96-trial block containing intermixed related and unrelated pairs of all transitive relations $\mathrm{BD}, \mathrm{DB}, \mathrm{CD}$, and DC, which were not tested with the MTS procedures. As in the previous block, while 48 trials presented pairs of related stimuli (e.g., C1D1), the other 48 trials displayed unrelated stimulus pairs (e.g., D1C2).

The semantic judgment trial started with the presentation of a white screen for $2,000 \mathrm{~ms}$. Thereafter, a fixation point (i.e., a cross) was shown at the center of the screen for $500 \mathrm{~ms}$, followed by a white screen displayed for $500 \mathrm{~ms}$, and the presentation of the prime stimulus (e.g., D1) at the center of the screen for $500 \mathrm{~ms}$. Next, the white screen was shown again for $600 \mathrm{~ms}$, followed by the target stimulus (e.g., C1) presented at the center of the screen for $500 \mathrm{~ms}$, and the presentation of the white screen for $500 \mathrm{~ms}$. Finally, a question mark was displayed in the center of the screen for $1,800 \mathrm{~ms}$, during which all participants had to respond to whether the prime and the target stimuli were related (or not) based on their prior experience with the MTS procedures. Participants were instructed to press the "S" key or the "N" key of the keyboard to indicate "related" or "not related," respectively. The responses were not followed by any feedback. All trials were separated by an ITI of $500 \mathrm{~ms}$ in which only the white screen was visible.

ERP recordings and statistical analysis All EEG recordings were obtained from seven electrodes placed on standardized locations (i.e., FZ, CZ, C3, C4, PZ, P3, and P4), according to the international 10-20 system. All electrodes were referenced to the linked mastoids and their impedance was below $5 \mathrm{k} \Omega$. Data were digitized at a rate of $1000 \mathrm{~Hz}$ and were processed using EEGLab (Version 14.0) and ERPLab (Version 6.1.3) toolbox software. The analysis was done at an epoch length of 1,000 ms, with $200 \mathrm{~ms}$ prestimulus baseline correction to $800 \mathrm{~ms}$ poststimulus onset. The trials in which the EEG activity exceeded $\pm 100 \mathrm{mV}$ were excluded.

A repeated-measure, two-tailed permutation test based on the $t_{\max }$ statistic (Blair \& Karniski, 1993) with a family-wise alpha level of 0.05 was used to analyze the differences between related and unrelated targets for each group. The $t_{\max }$ permutation test analysis was employed in lieu of the more conventional mean amplitude ANOVA as it provides an improved spatial and temporal resolution while maintaining an optimal family-wise alpha level due to the corrections following a large number of comparisons. Moreover, the $t_{\max }$ permutation test was reported to have relatively good power for ERP data with highly correlated dimensions (Hemmelmann, Horn, Reiterer, Schack, Susse, \& Weiss, 2004). To estimate the distribution of the null hypothesis, 2500 permutations were conducted, which were over twice the number recommended by Manly (1997) for a family-wise alpha level of 0.05 .

\section{Results}

\section{Baseline training and equivalence test}

The percentage of correct responses throughout the experiment for both the extended and reduced groups are shown in Table 1. In the AB training, 11 of 15 participants assigned to 
Table 1 Percentage of correct responses of the participants in both the extended and reduced group throughout all the phases of the experiment

\begin{tabular}{llllllll}
\hline Participants & AB & AC & CB I & AD & CB II & CB III & Test \\
\hline Extended & & & & & & & \\
P01 & $98 \%$ & $100 \%$ & $98 \%$ & $98 \%$ & $99 \%$ & $100 \%$ & $100 \%$ \\
P02 & $73 \%$ & $97 \%$ & $88 \%$ & $95 \%$ & $97 \%$ & $96 \%$ & $96 \%$ \\
P03 & $85 \%$ & $100 \%$ & $100 \%$ & $98 \%$ & $100 \%$ & $100 \%$ & $100 \%$ \\
P04 & $88 \%$ & $98 \%$ & $100 \%$ & $100 \%$ & $96 \%$ & $99 \%$ & $100 \%$ \\
P05 & $72 \%$ & $95 \%$ & $98 \%$ & $100 \%$ & $99 \%$ & $100 \%$ & $100 \%$ \\
P06 & $93 \%$ & $100 \%$ & $98 \%$ & $98 \%$ & $99 \%$ & $99 \%$ & $92 \%$ \\
P07 & $100 \%$ & $100 \%$ & $100 \%$ & $100 \%$ & $99 \%$ & $100 \%$ & $100 \%$ \\
P08 & $88 \%$ & $93 \%$ & $96 \%$ & $100 \%$ & $100 \%$ & $100 \%$ & $100 \%$ \\
P09 & $100 \%$ & $100 \%$ & $98 \%$ & $98 \%$ & $100 \%$ & $100 \%$ & $100 \%$ \\
P10 & $85 \%$ & $100 \%$ & $100 \%$ & $100 \%$ & $100 \%$ & $99 \%$ & $100 \%$ \\
P11 & $97 \%$ & $100 \%$ & $100 \%$ & $100 \%$ & $100 \%$ & $100 \%$ & $96 \%$ \\
P12 & $77 \%$ & $100 \%$ & $100 \%$ & $100 \%$ & $100 \%$ & $100 \%$ & $96 \%$ \\
P13 & $73 \%$ & $98 \%$ & $94 \%$ & $98 \%$ & $100 \%$ & $99 \%$ & $100 \%$ \\
P14 & $83 \%$ & $98 \%$ & $98 \%$ & $100 \%$ & $100 \%$ & $100 \%$ & $100 \%$ \\
P15 & $95 \%$ & $100 \%$ & $98 \%$ & $98 \%$ & $97 \%$ & $97 \%$ & $100 \%$ \\
Reduced & & & & & & & \\
P16 & $92 \%$ & $100 \%$ & $96 \%$ & $97 \%$ & $94 \%$ & $100 \%$ & $100 \%$ \\
P17 & $67 \%$ & $97 \%$ & $83 \%$ & $100 \%$ & $94 \%$ & $100 \%$ & $96 \%$ \\
P18 & $100 \%$ & $100 \%$ & $96 \%$ & $100 \%$ & $100 \%$ & $100 \%$ & $100 \%$ \\
P19 & $81 \%$ & $89 \%$ & $100 \%$ & $89 \%$ & $100 \%$ & $100 \%$ & $100 \%$ \\
P20 & $56 \%$ & $100 \%$ & $96 \%$ & $100 \%$ & $100 \%$ & $97 \%$ & $96 \%$ \\
P21 & $78 \%$ & $92 \%$ & $79 \%$ & $89 \%$ & $94 \%$ & $97 \%$ & $100 \%$ \\
P22 & $100 \%$ & $100 \%$ & $96 \%$ & $97 \%$ & $98 \%$ & $100 \%$ & $100 \%$ \\
P23 & $83 \%$ & $100 \%$ & $92 \%$ & $97 \%$ & $98 \%$ & $100 \%$ & $100 \%$ \\
P24 & $75 \%$ & $100 \%$ & $96 \%$ & $100 \%$ & $100 \%$ & $100 \%$ & $100 \%$ \\
P25 & $86 \%$ & $94 \%$ & $96 \%$ & $100 \%$ & $94 \%$ & $100 \%$ & $100 \%$ \\
P26 & $81 \%$ & $100 \%$ & $92 \%$ & $100 \%$ & $100 \%$ & $100 \%$ & $100 \%$ \\
P27 & $75 \%$ & $86 \%$ & $88 \%$ & $100 \%$ & $98 \%$ & $97 \%$ & $100 \%$ \\
P28 & $92 \%$ & $100 \%$ & $100 \%$ & $97 \%$ & $100 \%$ & $97 \%$ & $100 \%$ \\
P29 & $100 \%$ & $100 \%$ & $100 \%$ & $100 \%$ & $98 \%$ & $97 \%$ & $100 \%$ \\
P30 & $97 \%$ & $89 \%$ & $83 \%$ & $100 \%$ & $100 \%$ & $100 \%$ & $100 \%$ \\
\hline & & & & & & &
\end{tabular}

the extended group and 10 of 15 participants assigned to the reduced group reached the percentage of correct responses equal to or greater than $80 \%$. Considering the number of training trials for each group, $80 \%$ of the correct responses corresponded to 48 and 29 trials for the extended and the reduced groups, respectively.

While the participants in the extended group reached at least 93\% (56 of 60 training trials) of correct responses in the AC training, the participants assigned to the reduced group reached at least $86 \%$ (31 of 36 training trials) of correct responses. In the $\mathrm{AD}$ training, participants from the extended group reached equal to or greater than $95 \%$ of the correct choices (57 of 60 trials), whereas the participants assigned to the reduced group performed at or above $89 \%$ of correct choices (32 of 36 trials).
Table 2 Percentage of correct responses in the semantic judgment task for the extended and reduced group

\begin{tabular}{ccccc}
\hline \multicolumn{2}{c}{ Extended group } & & \multicolumn{2}{c}{ Reduced group } \\
\cline { 1 - 1 } Participants & \% correct responses & & Participants & $\%$ correct responses \\
\hline P1 & $100 \%$ & & P16 & $71 \%$ \\
P2 & $98 \%$ & & P17 & $98 \%$ \\
P3 & $97 \%$ & & P18 & $96 \%$ \\
P4 & $97 \%$ & & P19 & $93 \%$ \\
P5 & $100 \%$ & & P20 & $100 \%$ \\
P6 & $81 \%$ & & P21 & $98 \%$ \\
P7 & $100 \%$ & & P22 & $97 \%$ \\
P8 & $95 \%$ & & P23 & $98 \%$ \\
P9 & $100 \%$ & & P24 & $100 \%$ \\
P10 & $98 \%$ & & P25 & $94 \%$ \\
P11 & $97 \%$ & & P26 & $99 \%$ \\
P12 & $98 \%$ & & P27 & $77 \%$ \\
P13 & $100 \%$ & & P28 & $99 \%$ \\
P14 & $98 \%$ & & P29 & $100 \%$ \\
P15 & $98 \%$ & & P30 & $96 \%$ \\
\hline
\end{tabular}

Participants in both groups achieved high levels of success (90\% or above of correct responses) in most of the sessions, with the exception of four participants in the reduced group and one participant in the extended group who had a poorer performance in the Cumulative Baseline I. Finally, in the equivalence tests, only two participants in the reduced group and four participants in the extended group did not achieve $100 \%$ accuracy. Based on these data, we argue that participants' performance was substantially similar regardless of the group assigned during the training phase.

\section{Behavioral response on semantic judgment task}

The percentage of correct responses to semantic judgment tasks can be seen in Table 2. Correct responses in this task involved pressing the "S" key whenever a related stimulus pair (i.e., B1-D1) was presented and pressing the N-key whenever an unrelated pair of stimuli (i.e., B1-D2) was presented. Only trials for $\mathrm{BD}, \mathrm{DB}, \mathrm{CD}$, and $\mathrm{DC}$ relations were considered for this analysis. Fourteen of 15 participants assigned to the extended group indicated above $90 \%$ of correct responses, while 13 of 15 participants in the reduced group had a similar success rate. Once again, the participants' performance was very similar, regardless of the group assigned during the training phase.

\section{ERP recording analysis}

Since the trials in which the EEG activity exceeded $\pm 100 \mathrm{mV}$ were excluded from our analysis, the data from four 


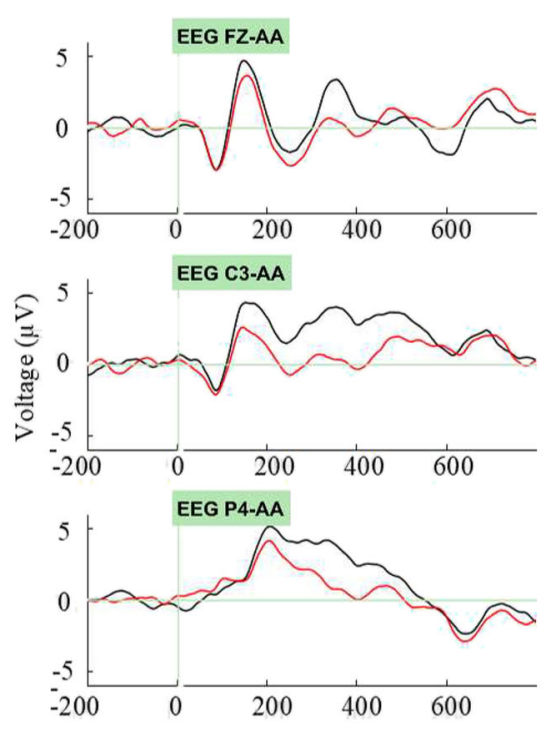

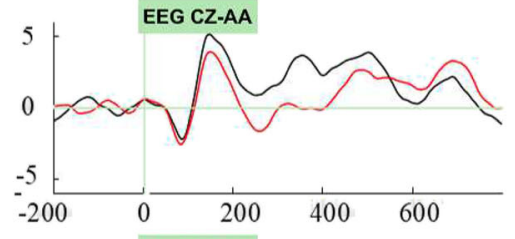

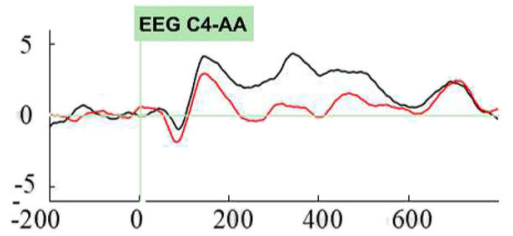

Related Stimulus Pairs
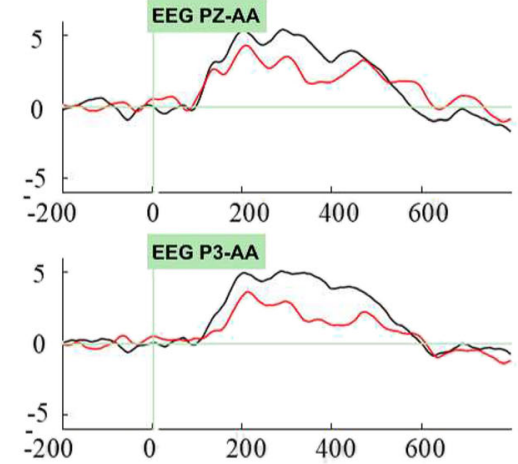

Fig. 2 Grand-average ERP waveforms for the participants in the reduced group when faced with stimulus pairs from the $\mathrm{CD}, \mathrm{DC}, \mathrm{BD}$, and $\mathrm{DB}$ emergent conditional relations. The black waveforms correspond to the trials labeled as "related," whereas the red waveforms correspond to the trials labeled as "unrelated." Each of the seven electrodes on the participant scalp were represented by a different chart. F, C, and P

participants in the reduced group (i.e., P19, P21, P26, and P28) were excluded from the ERP analysis, such that the analysis included 11 participants from the reduced group and 15 participants from the extended group.

Figure 2 shows the grand-average ERP waveforms for related targets (i.e., same class stimulus pair) in black and unrelated targets (i.e., different classes stimulus pair) in red, for the reduced group participants. Although visual inspection indicated reduced negativity between $300 \mathrm{~ms}$ and $500 \mathrm{~ms}$ for both the related and unrelated targets, the waveforms recorded for unrelated targets were systematically more negative than those recorded for related targets in that epoch.

The $t_{\text {max }}$ permutation test was used to detect reliable differences between the ERPs evoked by related and unrelated targets in the lexical decision tasks for the reduced group. All time points between $100 \mathrm{~ms}$ and $799 \mathrm{~ms}$ at the seven electrodes, which amounted to a total of 4,900 comparisons, were included in the test. A repeated-measures $t$ test was performed for each comparison using both the original data and the 2,500 random within-participant permutations. The maximum $t$ score $\left(t_{\text {max }}\right)$ was recorded in each of the 2,501 sets of tests and used to estimate the $t_{\max }$ distribution of the null hypothesis (i.e., no difference between conditions). The null hypothesis of the permutation test states that positive differences between conditions are just as likely to have been negative differences and vice versa. Therefore, the distribution of the null hypothesis is symmetric around a difference of zero.

Based on this estimation, critical $t$ scores of $\pm 6.216(d f=10)$ were derived for the reduced group such that any differences in the original data that exceeded a $t$ score of \pm 6.216 letters refer to the frontal, central, and parietal regions, respectively, whereas the $\mathrm{Z}$ letter refers to the midline sagittal plane of the participant skull. Even numbers indicate locations on the right hemisphere, and odd numbers refer to the left hemisphere. The AA letters indicate that the electrodes were referred to the left and right mastoid of each participant. (Color figure online)

were deemed reliable. Reliable differences between the ERPs evoked by the different classes and from withinclass were observed from $333 \mathrm{~ms}$ to $360 \mathrm{~ms}$ in C3, P3, $\mathrm{C} 4$, and P4 electrodes (see Fig. 3, raster plot) for participants in the reduced group.

Similar to Fig. 2, Fig. 4 presents the grand-average ERP waveforms for the participants in the extended group when exposed to stimulus pairs from the CD, DC, BD, and DB emergent conditional relations. Black lines correspond to the related targets whereas the red lines indicate unrelated targets. Visual inspection of the data suggested reduced negativity between $300 \mathrm{~ms}$ and $500 \mathrm{~ms}$ in the waveforms of both related and unrelated targets, except for the records obtained from the FZ locus, in which the wave amplitudes were considerably dissimilar for both trial types.

The ERP records were submitted to a repeated-measure, two-tailed permutation test based on the $t_{\max }$ statistic (Blair $\&$ Karniski, 1993) using a family-wise alpha level of 0.05 . Critical $t$ scores of $\pm 5.104(d f=14)$ were derived for the extended group (i.e., any differences in the original data that exceeded a $t$ score of \pm 5.104 for the extended group were deemed reliable). In this case, however, an absence of reliable differences between the ERPs evoked by a stimulus from different classes and from the same class was observed for participants in the extended group.

\section{Discussion}

The present study evaluated whether prior exposure to different numbers of baseline training trials could modulate the 


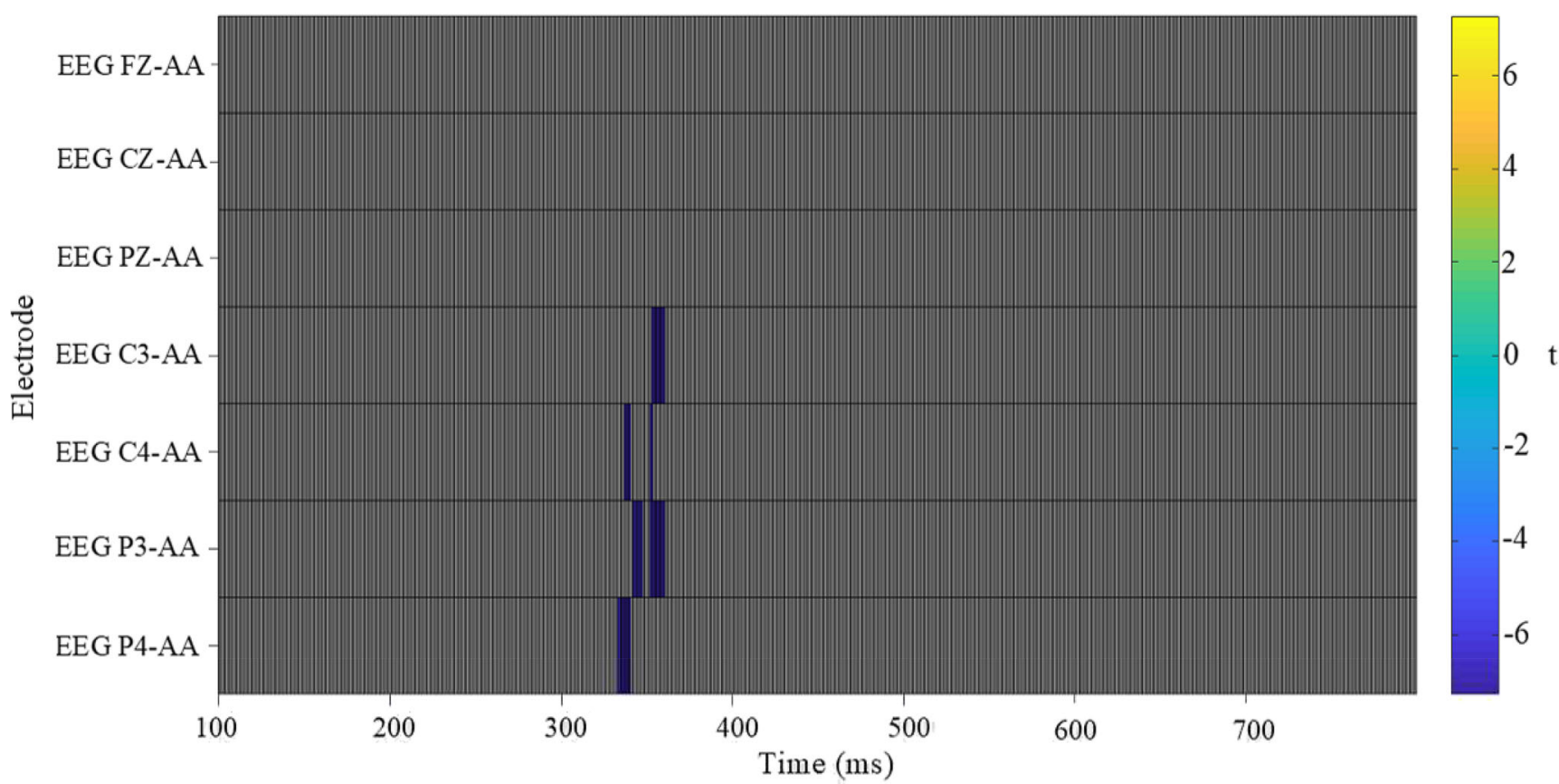

Fig. 3 Raster diagram of the permutation test for the reduced group, with each electrode represented in a row and each time point represented by a column. Each box in the diagram therefore represents the result of a $t$ test. Colored boxes indicate that the difference wave is significantly negative at that time point and electrode (even after effectively correcting for multiple comparisons). Gray boxes indicate that the $t$ test is not significant (after effectively correcting for multiple comparisons). (Color figure online)
N400 component. To this end, participants in the extended group performed approximately twice as many trials as participants in the reduced group. Our findings suggest that overexposure to training trials evoked lower negativity in the N400 component for participants in the extended group. The EEG had very similar waveforms for related and unrelated stimulus pairs around the 400-ms epochs (see Fig. 4). In contrast, exposure to a lower number of training trials evoked higher negativity in the $\mathrm{N} 400$ component among participants in the reduced group, who had larger negative-going wave for unrelated stimulus pairs compared to the ones for related stimulus pairs (see Figs. 2 and 3).
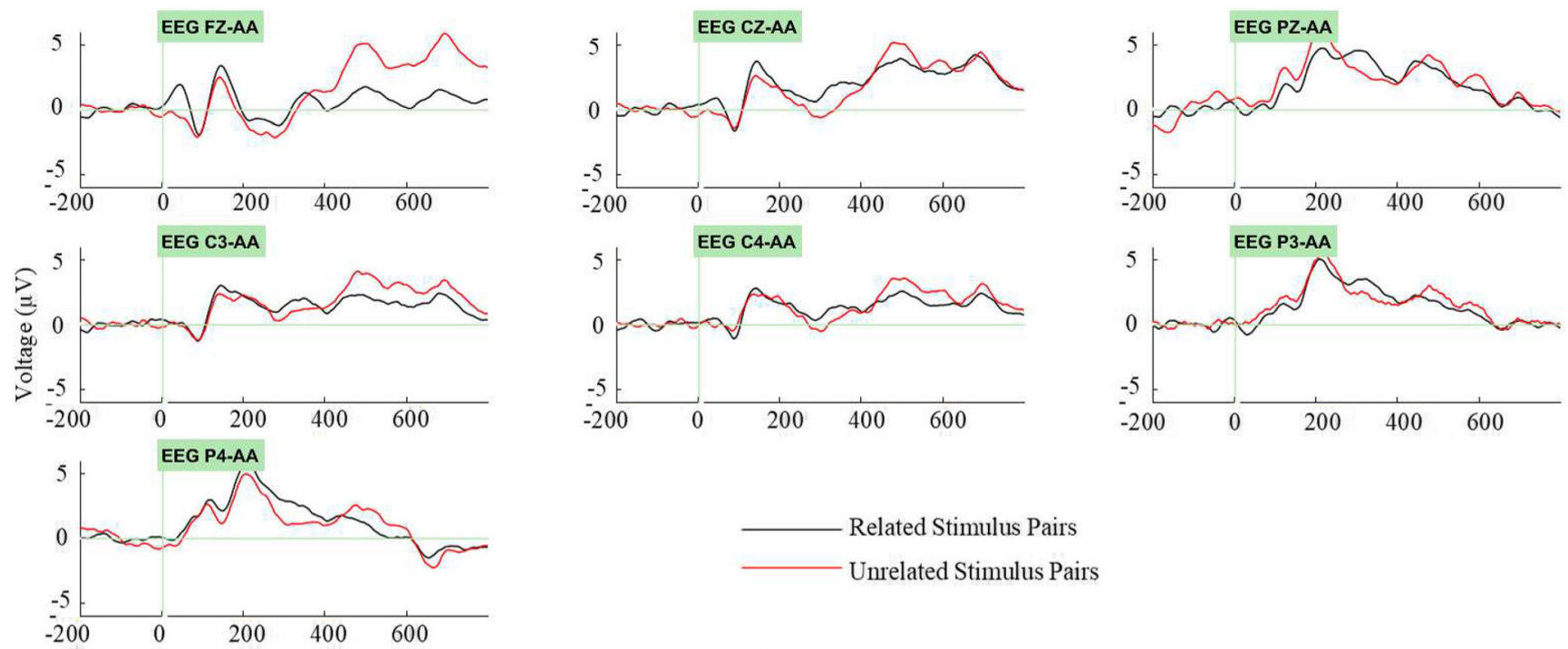

Time (ms)

Fig. 4 Grand-average ERP waveforms for the participants in the extended group when faced with stimulus pairs from the CD, DC, BD, and DB emergent conditional relations. The black waveforms correspond to the trials labeled as "related," whereas the red waveforms correspond to the trials labeled as "unrelated." (Color figure online) 
Interestingly, while a higher number of training trials improved performance on equivalence tests (Espírito-Santo et al., 2019; Straatmann et al., 2014), our findings suggest that they play an opposite role as regards to the N400 component. In reference to experiments that relate equivalence class formation to the N400 component, we argue that the number of trials performed by participants during baseline training should be analyzed more carefully. As stated above, experimental protocols, in general, only establish learning criteria for some of the training blocks; however, the participants are exposed to as many training blocks as necessary until they are fully trained. For instance, the learning criterion can be achieved after a single exposure or after three or four exposures to the training block. If the N400 component is recorded in this context, the effect of the investigated variable can be confused with the effect of the number of trials performed by them.

For participants exposed to a total of 96 trials with four conditional relations (Tabullo et al., 2015a, 2015b), 24 trials of each of the conditional relation were administered prior to the semantic judgment task. N400 waveforms evoked for related pairs in this case were different from the ones for unrelated pairs. Consistent with these findings, participants in the reduced group also performed 24 trials of each conditional relation trained during the baseline, and analogous evidence regarding the N400 component was observed. A similar number of trials was also used by Wang and Dymond (2013, Experiment 2), in which the participants were exposed to approximately 25 trials of each conditional relation. However, no difference in the N400 component was observed in the semantic judgment task. Finally, participants in the D. Barnes-Holmes et al. (2005) study performed an average of 398 baseline training trials. The training was done on six conditional relations, thereby exposing the participants to approximately 66 trials of each conditional relation. Despite the number being considerably higher than the 44 programmed trials for participants in the extended group, robust N400 component differences were reported for pairs of related and unrelated stimuli.

A more extensive comparative analysis of the results described in the present experiment, and those described in previous studies that relate equivalence class formation to the N400 component, was hindered due to the lack of information regarding the number of completed trials. In this specific set of experiments, the number of trials planned in the training phase and the learning criteria applied to each phase were reported; however, information regarding the actual number of trials was absent in a majority of previous cases. The differences in the number of trials, along with differences in other experimental parameters may explain the inconsistencies in the results. Our findings show that with all other parameters constant, increasing the number of training trials negatively affects the N400 component.

Although the current study is designed to describe the independent modulating effect of each parameter used in the equivalence class, future studies are needed to develop protocols to investigate the relative modulatory effect of various parameters on the N400 component. For instance, it can be argued that the unfavorable bias related to the large number of training trials may have been mitigated by previous MTS tests in the D. Barnes-Holmes et al. (2005) experiment. The parametric evaluations, as suggested here, refer to a considerably extensive research program involving multiple experiments; however, considering that the recent studies aimed at determining equivalence relations with the $\mathrm{N} 400$ effect have provided encouraging results, it seems a worthwhile effort.

\section{Equivalence relations and $\mathrm{N} 400$ using abstract figures as stimuli}

To our knowledge, the present study is the first to demonstrate the N400 effect using abstract figures as stimuli in an equivalence class formation experiment. Yorio et al. (2008) and Wang and Dymond (2013, Experiment 2) used training procedures composed only of abstract figures, but the results for the N400 effects were not clear. Of the six previous studies with positive results for the N400 component, three used pseudowords as stimuli (D. Barnes-Holmes et al., 2005; Tabullo et al., 2015a, b), two used abstract and meaningful figures in the baseline training (Bortoloti et al., 2014; Granerud-Dunvoll et al., 2019), and one used nonsense words and abstract figures as stimuli (Haimson et al., 2009). Our finding is noteworthy because pairs of abstract figures, presumably without preexperimental meaning, produce a temporal pattern of neural activity similar to that observed for natural language words. These results suggest that the stimulus equivalence paradigm may provide a useful experimental model for studying semantic relations and indicate that additional studies should be performed to investigate the modulating effects of different types of stimuli (i.e., abstract figures, pseudowords, and meaningful figures) on the N400 component.

\section{The semantic judgment task as an index for equivalence relations}

In the current study, $\mathrm{BC}$ and $\mathrm{CB}$ emergent relations were evaluated using MTS tests, while $\mathrm{BD}, \mathrm{DB}, \mathrm{CD}$, and $\mathrm{DC}$ emergent relations were evaluated using the semantic judgment task. Considering the differences between tasks, semantic judgment provides an additional index for equivalence class formation by requiring participants to classify the stimulus pairs as being related or unrelated. Thus, classifying B1-D1 as a pair of related stimuli could indicate that $\mathrm{B} 1$ and D1 were members of the same class, while classifying B1-D2 as a pair of unrelated stimuli could indicate that they were in different classes. Given that almost all participants of both groups achieved a percentage of correct responses higher than $90 \%$ in this task, it is possible to argue that $\mathrm{D}$ stimuli were part of the equivalence classes. These findings are noteworthy in the present 
experiment because they allow us to reject the possibility that the differences in the N400 component between groups are due to the noninclusion of the $\mathrm{D}$ stimuli in the classes (i.e., D stimuli were not part of the classes for the majority of the participants in extended group leading to a lower negativity in the range of the N400 component).

As an index for equivalence class formation, the semantic judgment tasks can be compared with the stimulus sorting tests, an alternative procedure for evaluating equivalence classes (Arntzen et al., 2017; Fields, Arntzen, \& Moksness, 2014; Fields, Arntzen, Nartey, \& Eilifsen, 2012). In the sorting test, equivalence class formation is evaluated through a deck of cards. Specifically, each card contains one of the stimuli used in previous baseline training. Participants are required to arrange shuffled decks into groups. If the group has the same stimuli as in the equivalence class, it is considered a successful secondary measure of class formation. According to Fields et al. (2014), in some cases, sorting tests appeared to be more sensitive to tracking class formation, faster, and easier to interpret, than the MTS tests. Therefore, sorting may be a useful alternative for evaluating equivalence class formation. The aspects applicable for sorting tests could also be true for semantic judgment tasks, but further research is needed to verify that.

\section{Comparing psycholinguistic and equivalence class formation findings}

Our findings can be related to an issue frequently referred to in the equivalence class literature-namely, the relatedness of the stimuli in the same equivalence class. Some conditional relations between the stimuli in the same class are more strongly established in comparison with the other conditional relations (e.g., Bortoloti \& de Rose, 2012; Bortoloti et al., 2013; Doran \& Fields, 2012; Fields, Adams, Verhave, \& Newman, 1993; Moss-Lourenco \& Fields, 2011). Bortoloti et al. (2013), for instance, used a semantic differential device and showed that overtraining of baseline relations resulted in improved transfer outcomes from pictures portraying happiness and anger to abstract stimuli as compared to the transfer outcomes after a reduced training of baseline relations. This indicated that equivalence relations composed of overtrained baseline relations were more strongly established than the classes formed by fewer training trials. Therefore, the greater number of training trials for the participants in the extended group presumably engendered stronger relationships between the members of the equivalence class.

Interestingly, previous studies on language processing have reported that the modulation of the N400 was associated with the level of semantic information present in the word pairs. Specifically, the greater the semantic relationship between words in naturally occurring language, the more they were predictable and easier to process. This can result in the reduction of the N400 wavelengths (Kutas \& Federmeier, 2000). In the present experiment, the greater number of baseline training trials performed by extended group participants established stronger conditional relations between stimuli as compared with those performed by the reduced group participants. In accordance with Kutas and Federmeier (2000), our findings showed that the N400 effect was weaker for the extended group than for the reduced group.

Our findings were also related to the N400 effect described in the experiments on language processing that evaluated the effect of repeated word presentation (e.g., Bentin \& Peled, 1990; Deacon, Dynowska, Ritter, \& Grose-Fifer, 2004; Laszlo, Stites, \& Federmeier, 2012; Rugg, 1990; Rugg, Doyle, \& Melan, 1993; Smith \& Halgren, 1987; Van Petten, Kutas, Kluender, Mitchiner, \& McIsaac, 1991). Smith and Halgren (1987), for example, described the ERPs evoked by repetitions of highfrequency and low-frequency words from participants' native language. In their experiment, two blocks of words were presented in lexical decision tasks. The initial block consisted of a unique presentation of high-frequency and low-frequency words, whereas the second block contained multiple repetitions of words from the initial block in addition to unique presentations of new words. Interestingly, the exceedingly repeated words in the second block evoked a less prominent N400 than the nonrepeated words. Regarding the equivalence class literature, Granerud-Dunvoll et al. (2019, Experiment 1) conducted an experiment in which abstract and meaningful figures were related in the MTS protocol. After the equivalence tests, participants were given two blocks of semantic judgment tasks, both composed of related and unrelated stimulus pairs. Differences in the N400 component were obtained when the waveforms for the unrelated stimulus pairs from the first block were compared to the waveforms for the same unrelated stimulus pairs from the second block. The authors point out that the repetition of the stimuli throughout the semantic judgment task may have weakened the N400 component. In summary, the repetition effect reported by Smith and Halgren in lexical decision tasks with words from participants' native language appears to be similar to the repetition effect described by Granerud-Dunvoll et al. in priming tasks with conditional relations of the equivalence classes.

Smith and Halgren (1987) also reported that more prominent N400 wavelengths were evoked by low-frequency words than by the high-frequency words. Although the effects of word frequency in a natural language cannot be controlled in the laboratory with the current experimental methods, it is possible that the manipulation of the number of baseline trials during the training phase can simulate the word frequency effect to some degree. If true, the N400 effect described in 
the extended group participants can be compared with that described for high-frequency words and the N400 effect described in the reduced group participants can be compared with that described for low-frequency words. Once again, our findings are in line with those described in the cognitive literature, showing that the low-frequency words and fewer baseline training trials positively affect the N400 component.

\section{Concluding remarks}

The current study provides a more comprehensive understanding of how the number of baseline training trials influences the N400 component. The results of the present experiment indicate the necessity for a more measured approach in deciding the number of baseline training trials used in experiments relating to equivalence relations and the N400 component. In addition, considering the similarities between our findings and those regarding the repetition effect of high and low frequency words (Kutas \& Federmeyer, 2000; Rugg, 1990; Smith \& Halgren, 1987), our results provide additional evidence that stimulus equivalence (Sidman, 1971, 1994, 2000) can be an experimental analog of semantic processing by grasping the nuances that characterize the human language.

Author note This manuscript is based on a thesis submitted by Ryan Espírito-Santo in partial fulfillment of the requirements for a master's degree in Psychology at the Universidade Federal de Minas Gerais.

Edson Huziwara was supported by Conselho Nacional de Ciência e Tecnologia (CNPq, Grant No. 472407/2013-9). This research was supported by the National Institute of Science and Technology on Behavior, Cognition, and Teaching (INCT-ECCE 1), supported by FAPESP (Grant No. 2008/57705-8) and CNPq (Grant No. 573972/2008-7), 2009-2016.

All authors are affiliated with the National Institute of Science and Technology on Behavior, Cognition, and Teaching (INCT - ECCE 2), supported by FAPESP (Grant No. 2014/50909-8), CAPES (Grant No. 88887.136407/2017-00), and CNPq (Grant No. 465686/2014-1).

Compliance with ethical standards This manuscript has not been published or presented elsewhere, in part or in its entirety, and is not under consideration by another journal.

Conflict of interest There are no conflicts of interest to declare.

Ethical approval All procedures performed involving human participants were in accordance with the ethical standards of the institutional and/or national research committee, as well as with the 1964 Helsinki declaration and its later amendments or comparable ethical standards.

Informed consent Proper informed consent was obtained, and the study design was approved by the appropriate ethics review boards. All the authors have approved the manuscript and agree with the submission to your esteemed journal.

Open practices statements None of the data or materials for the experiments reported here are available, and none of the experiments were preregistered.

\section{References}

Almeida-Verdu, A. C. M., Huziwara, E. M., de Souza, D. G., de Rose, J. C., Bevilacqua, M. C., Lopes, J. Jr., . . Mcllvane, W. J. (2008). Relational learning in children with deafness and cochlear implants. Journal of the Experimental Analysis of Behavior, 89, 407-424. https://doi.org/10.1901/jeab.2008-89-407

Arntzen, E. (2012). Training and testing parameters in formation of stimulus equivalence: Methodological issues. European Journal of Behavior Analysis, 13, 123-135. https://doi.org/10.1080/ 15021149.2012.11434412

Arntzen, E., Granmo, S., \& Fields, L. (2017). The relation between sorting tests and matching-to-sample tests in the formation of equivalence classes. The Psychological Record, 67, 1-16. https://doi.org/ 10.1007/s40732-016-0209-9

Barnes-Holmes, D., Stauton, C., Whelan, R., Barnes-Holmes, Y., Commins, S., Walsh, D., . . Dymond, S. (2005). Derived stimulus relations, semantic priming, and event-related potentials: Testing a behavioral theory of semantic networks. Journal of the Experimental Analysis of Behavior, 84, 417-433. https://doi.org/ 10.1901/jeab.2005.78-04

Barnes-Holmes, Y., Barnes-Holmes, D., Smeets, P. M., \& Luciano, C. (2004). A derived transfer of mood functions through equivalence relations. The Psychological Record, 54, 95-113. https://doi.org/10. 1007/BF03395464

Bentin, S., \& Peled, B. (1990). The contribution of task-related factors to ERP repetition effects at short and long lags. Memory \& Cognition, 18, 359-366. https://doi.org/10.3758/BF03197125

Blair, R. C., \& Karniski, W. (1993). An alternative method for significance testing of waveform difference potentials. Psychophysiology, 30, 518-524. https://doi.org/10.1111/j.1469-8986.1993.tb02075.x

Bortoloti, R., \& de Rose, J. C. (2007). Medida do grau de relacionamento entre estímulos equivalentes [Measuring the relatedness of equivalent stimuli.] Psicologia: Reflexão e Crítica, 20, 252258. https://doi.org/10.1590/S0102-79722007000200011

Bortoloti, R., \& de Rose, J. C. (2009). Assessment of the relatedness of equivalent stimuli through a semantic differential. The Psychological Record, 59, 563-590. https://doi.org/10.1007/ BF03395682

Bortoloti, R., \& de Rose, J. C. (2011). Avaliação do efeito de dica semântica e da indução de significado entre estímulos abstratos equivalentes [Evaluation of semantic priming effect and induction of meaning among abstract equivalent stimuli]. Psicologia: Reflexão e Crítica, 24, 381-393. https://doi.org/ 10.1590/S0102-79722011000200020

Bortoloti, R., \& de Rose, J. C. (2012). Equivalent stimuli are more strongly related after training with delayed matching than after simultaneous matching: A study using the implicit relational assessment procedure (IRAP). The Psychological Record, 62, 4154. https://doi.org/10.1007/BF03395785

Bortoloti, R., Pimentel, N., \& de Rose, J. C. (2014). Electrophysiological investigation of the functional overlap between semantic and equivalence relations. Psychology \& Neuroscience, 7, 183-191. https:// doi.org/10.3922/j.psns.2014.015

Bortoloti, R., Rodrigues, N. C., Cortez, M. D., Pimentel, N., \& de Rose, J. C. (2013). Overtraining increases the strength of equivalence relations. Psychology \& Neuroscience, 6, 357-364. https://doi.org/10. 3922/j.psns.2013.3.13

Capobianco, D., \& Picanço, C. R. F. (2015). EAM-Emparelhamento ao modelo (Programa de computador Delphi) [Computer software]. Retrieved from https://github.com/eep-lab/eam_docs/releases

de Rose, J. C., de Souza, D. G., Aiello, A. L., \& de Rose, T. M. S. (1992). Stimulus equivalence and generalization in reading after matching to sample by exclusion. In S. C. Hayes \& L. J. 
Hayes (Eds.), Understanding verbal relations (pp. 69-82). Reno, NV: Context Press.

Deacon, D., Dynowska, A., Ritter, W., \& Grose-Fifer, J. (2004). Repetition and semantic priming of nonwords: Implications for the theories of N400 and word recognition. Psychophysiology, 41, 60 74. https://doi.org/10.1111/1469-8986.00120

Debert, P., Matos, M. A., \& McIlvane, W. J. (2007). Conditional relations with compound abstract stimuli using a go/no-go procedure. Journal of the Experimental Analysis of Behavior, 87, 89-96. https://doi.org/ 10.1901/jeab.2007.46-05

Doran, E., \& Fields, L. (2012). All stimuli are equal, but some are more equal than others: Measuring relational preferences within an equivalence class. Journal of the Experimental Analysis of Behavior, 98, 243-256. https://doi.org/10.1901/jeab.2012.98-243

Espírito-Santo, R. R. B., Rico, V. V., \& Huziwara, E. M. (2019). Efeitos do overtraining na formação de classes de estímulos equivalentes em estudantes universitários [Effects of overtraining on the formation of stimulus equivalence classes in college students]. Acta Comportamentalia, 27, 55-72.

Fields, L., Adams, B. J., Verhave, T., \& Newman, S. (1993). Are stimuli in equivalence classes equally related to each other. The Psychological Record, 46, 85-105.

Fields, L., Arntzen, E., \& Moksness, M. (2014). Stimulus sorting: A quick and sensitive index of equivalence class formation. The Psychological Record, 64, 487-498. https://doi.org/10.1007/ s40732-014-0034-y

Fields, L., Arntzen, E., Nartey, R. K., \& Eilifsen, C. (2012). Effects of a meaningful, a discriminative, and a meaningless stimulus on equivalence class formation. Journal of the Experimental Analysis of Behavior, 97, 163-181. https://doi.org/10.1901/jeab.2012.97-163

Granerud-Dunvoll, G., Arntzen, E., Elvsåshagen, T., Hatlestad-Hall, C., \& Malt, A. (2019). Equivalence class formation and the N400: Methodological issues. The Psychological Record, 69, 175-191. https://doi.org/10.1007/s40732-019-00332-x

Haimson, B., Wilkinson, K. M., Rosenquist, C., Ouimet, C., \& McIlvane, W. J. (2009). Electrophysiological correlates of stimulus equivalence processes. Journal of the Experimental Analysis of Behavior, 92, 245-256. https://doi.org/10.1901/jeab.2009.92-245

Hajra, S. G., Liu, C. C., Song, X., Fickling, S. D., Cheung, T. P. L., \& D'Arcy, R. C. N. (2018). Multimodal characterization of the semantic N400 response within a rapid evaluation brain vital sign framework. Journal of Translational Medicine, 16, 151. https://doi.org/ 10.1186/s12967-018-1527-2

Hemmelmann, C., Horn, M., Reiterer, S., Schack, B., Susse, T., \& Weiss, S. (2004). Multivariate tests for the evaluation of high-dimensional EEG data. Journal of Neuroscience Methods, 139, 111-120. https:// doi.org/10.1016/j.jneumeth.2004.04.013

Holcomb, P. J. (1993). Semantic priming and stimulus degradation: Implications for the role of the N400 in language processing. Psychophysiology, 30, 47-61. https://doi.org/10.1111/j.1469-8986. 1993.tb03204.x

Kutas, M., \& Federmeier, K. D. (2000). Electrophysiology reveals semantic memory use in language comprehension. Trends in Cognitive Science, 4, 463-470. https://doi.org/10.1016/S13646613(00)01560-6

Kutas, M., \& Federmeier, K. D. (2011). Thirty years and counting: Finding meaning in the N400 component of the event-related brain potential (ERP). Annual Review of Psychology, 62, 621-647. https:// doi.org/10.1146/annurev.psych.093008.131123

Kutas, M., \& Hillyard, S. A. (1984). Brain potentials during reading reflect word expectancy and semantic association. Nature, 307, 161-163. https://doi.org/10.1038/307161a0

Kutas, M., \& Hillyard, S. A. (1989). An electrophysiological probe of incidental semantic association. Journal of Cognitive Neuroscience, 1, 38-49. https://doi.org/10.1162/jocn.1989.1.1.38
Laszlo, S., Stites, M., \& Federmeier, K. D. (2012). Won’t get fooled again: An event-related potential study of task and repetition effects on the semantic processing of items without semantics. Language \& Cognitive Processes, 27, 257-274. https://doi.org/10.1080/ 01690965.2011.606667

Lau, E. F., Phillips, C., \& Poeppel, D. (2008). A cortical network for semantics: (De) constructing the N400. Nature Reviews Neuroscience, 9, 920-933. https://doi.org/10.1038/nrn2532

Leader, G., Barnes, D., \& Smeets, P. (1996). Establishing equivalence relations using a respondent-type training procedure. The Psychological Record, 46, 685-706.

Luck, S. J., \& Kappenman, E. S. (2011). The Oxford handbook of eventrelated potential components. New York, NY: Oxford University Press.

Manly, B. F. J. (1997). Randomization, bootstrap, and Monte Carlo methods in Biology (2nd ed.). London, UK: Chapman \& Hall.

Matos, M. A., Avanzi, A. L., \& McIlvane, W. J. (2006). Rudimentary reading repertoires via stimulus equivalence and recombination of minimal verbal units. The Analysis of Verbal Behavior, 22, 3-19. https://doi.org/10.1007/BF03393023

Menendéz, J., Sánchez, F., Polti, I., Idesis, S., Avellaneda, M., Tabullo, A., \& Iorio, A. (2018). Event-related potential correlates of stimulus equivalence classes: A study of task order of the equivalence based priming probes with respect to the stimulus equivalence tests, and among the distinct trial types with each other. Behavioural Brain Research, 347, 242-254. https://doi.org/10.1016/j.bbr.2018.03.017

Moreno, E., \& Kutas, M. (2005). Processing semantic anomalies in two languages: An electrophysiological exploration in both languages of Spanish-English bilinguals. Brain Research. Cognitive Brain Research, 22, 205-220. https://doi.org/10.1016/j. cogbrainres.2004.08.010

Moss-Lourenco, P., \& Fields L. (2011). Nodal structure and stimulus relatedness in equivalence classes: Post-class formation preference tests. Journal of the Experimental Analysis of Behavior, 95, 343368. https://doi.org/10.1901/jeab.2011.95-343

O'Toole, C., Barnes-Holmes, D., \& Smyth, S. (2007). A derived transfer of functions and the implicit association test. Journal of the Experimental Analysis of Behavior, 88, 263-283. https://doi.org/ 10.1901/jeab.2007.76-06

Rugg, M. D. (1990). Event-related brain potentials dissociate repetition effects of high- and low-frequency words. Memory \& Cognition, 18 , 367-379. https://doi.org/10.3758/BF03197126

Rugg, M. D., Doyle, M. C., \& Melan, C. (1993). An event-related potential study of the effects of within- and across-modality word repetition. Language \& Cognitive Processes, 8, 357-377. https://doi.org/ $10.1080 / 01690969308407582$

Saunders, R. R., \& McEntee, J. E. (2004). Increasing the probability of stimulus equivalence with adults with mild mental retardation. The Psychological Record, 54, 423-435. https://doi.org/10.1007/ BF03395483

Saunders, R. R., Wachter, J. A., \& Spradlin, J. E. (1988). Establishing auditory stimulus control over an eight members equivalence class via conditional discrimination procedures. Journal of the Experimental Analysis of Behavior, 49, 95-115. https://doi.org/10. 1901/jeab.1988.49-95

Sidman, M. (1971). Reading and auditory-visual equivalences. Journal of Speech and Hearing Research, 14, 5-13. https://doi.org/10.1044/ jshr. 1401.05

Sidman, M. (1994). Equivalence relations and behavior: A research history. Boston, MA: Authors Cooperative.

Sidman, M. (2000). Equivalence relations and the reinforcement contingency. Journal of the Experimental Analysis of Behavior, 74, 127 146. https://doi.org/10.1901/jeab.2000.74-127

Sidman, M., \& Tailby, W. (1982). Conditional discrimination vs. matching to sample: An expansion of the testing paradigm. 
Journal of the Experimental Analysis of Behavior, 31, 5-22. https:// doi.org/10.1901/jeab.1982.37-5

Silveira, M. V., Sbrocco, G., Sarmento, R., Cortez, M., Ramos, C., \& de Rose, J. (2019). The use of N400 in studies of stimulus equivalence: A review of methods and parameters. Revista Brasileira de Análise do Comportamento, 14(1). https://doi. org/10.18542/rebac.v14i1.7187

Smeets, P. M., \& Barnes-Holmes, D. (2003). Children's emergent preferences for soft drinks: Stimulus-equivalence and transfer. Journal of Economic Psychology, 24, 603-618. https://doi.org/10.1016/S01674870(03)00004-7

Smith, M. E., \& Halgren, E. (1987). Event-related potentials during lexical decision: Effects of repetition, word frequency, pronounceability, and concreteness. In R. Johnson, J. W. Rohrbaugh, \& R. Parasuraman (Eds.), Current trends in ERP research (pp. 417421). Amsterdam, Netherlands: Elsevier.

Straatmann, G., Almeida, S. S., \& de Rose, J. C. (2014). Computerized assessment of food preferences in adolescents in the stimulus equivalence paradigm. Temas em Psicologia, 22, 613-624. https://doi. org/10.9788/TP2014.3-07

Tabullo, A., Yorio, A., Zanutto, S., \& Wainselboin, A. (2015a). ERP correlates of priming in language and stimulus equivalence: Evidence of similar N400 effects in absence of semantic content. International Journal of Psychophysiology, 96, 74-83. https://doi. org/10.1016/j.ijpsycho.2015.03.004
Tabullo, A., Yorio, A., Zanutto, S., \& Wainselboin, A. (2015b). An ERP comparison of derived relations in stimulus equivalence classes. Psychology \& Neuroscience, 8, 509-528. https://doi.org/10.1037/ pne0000028

Tyndall, I. T., Roche, B., \& James, J. E. (2004). The relation between stimulus function and equivalence class formation. Journal of the Experimental Analysis of Behavior, 81, 257-266. https://doi.org/10. 1901/jeab.2004.81-257

Van Petten, C., Kutas, M., Kluender, R., Mitchiner, M., \& McIsaac, H. (1991). Fractionating the word repetition effect with event-related potentials. Journal of Cognitive Neuroscience, 3, 131-150. https:// doi.org/10.1162/jocn.1991.3.2.131

Wang, T., \& Dymond, S. (2013). Event-related potential correlates of emergent inference in human arbitrary relational learning. Behavioural Brain Research, 236, 332-343. https://doi.org/10. 1016/j.bbr.2012.08.033

Yorio, A., Tabullo, A., Wainselboim, A., Barttfeld, P., \& Segura, E. (2008). Event-related potential correlates of perceptual and functional categories: Comparison between stimuli matching by identity and equivalence. Neuroscience Letters, 443, 113-118. https://doi.org/10. 1016/j.neulet.2008.07.001

Publisher's note Springer Nature remains neutral with regard to jurisdictional claims in published maps and institutional affiliations. 